\title{
ISO 14006. Experiencias previas de estudios de arquitectura que han adoptado el estándar de ecodiseño UNE 150301:2003
}

\section{ISO 14006. Previous experiences in the adoption of the standard UNE 150301 by the architecture studios}

G. Arana-Landín ${ }^{(*)}$, E. Cilleruelo ${ }^{(*)}$, J. C. Aldasoro ${ }^{(*)}$

RESUMEN

En este artículo se analiza la experiencia de implantación de la norma UNE 150301 de ecodiseño en el sector de los estudios de arquitectura.

Tras el examen de la estructura de norma UNE 150301, de sus objetivos y de su alcance, se analiza su difusión, destacándose que el $73 \%$ de las empresas certificadas en España son estudios de arquitectura. A continuación se estudia el proceso de implantación real, mediante el estudio de caso que lo analiza en cinco estudios de arquitectura.

El estudio realizado permite conocer de primera mano las principales claves y dificultades del proceso de implantación del estándar, así como los resultados obtenidos, entre los que destaca una reducción del impacto ambiental. Se constata, en suma, que la norma UNE 150301 es una herramienta que puede proporcionar ventajas competitivas interesantes a las empresas del sector de los estudios de arquitectura.
SUMMARY

This paper aims to analyze the pioneer UNE 150301 standard, as well as its adoption process and its practical results in the sector of architecture studios.

First, the structure, the aim and the scope of norm UNE 150301 have been analysed. Second, the standard's implementation has been examined, concluding that $73 \%$ of the companies that have obtained the certificate are architecture studios. A case study has therefore been carried out with the participation of five architecture studios pioneers.

These experiences have let us know the main aspects and difficulties of the process. In conclusion, the adoption of the standard UNE 150301 can be a helpful tool in order to reduce the environmental impact of the products and obtain some competitive advantages such as cost reduction, improvement in energy efficiency of the product and a better adaptation to acts and regulations.

Keywords: UNE 150301; life cycle; environmental standard; ISO 14006; ecodesign.
Palabras clave: UNE 150301; ciclo de vida; estándar medioambiental; ISO 14006; ecodiseño.
Recibido/Received: 08 mar 2011 Aceptado/Accepted: 22 nov 2011 Publicado online/ Published online: 31 may 2012

(*) Escuela Politécnica-Universidad del País Vasco (UPV/EHU). San Sebastián (España). Persona de contacto/Corresponding author: g.arana@ehu.es (G. Arana-Landín). 


\section{INTRODUCCIÓN}

El ecodiseño considera los impactos ambientales, es decir, los cambios en el medioambiente total o parcialmente resultantes de las actividades, productos o servicios de una organización, en todas las etapas del proceso de diseño y desarrollo de productos, para lograr que generen el mínimo impacto ambiental a lo largo de todo su ciclo de vida. Éste está compuesto por todas las etapas consecutivas e interrelacionadas de un sistema del producto, desde la adquisición de materia prima o de su generación a partir de recursos naturales, hasta la disposición final (1).

Las teorías actuales de ecodiseño subrayan que la función del diseñador resulta clave en todo lo concerniente al ecodiseño, debido a que desarrolla su trabajo en las fases incipientes del proceso de desarrollo del producto (2). Por estos motivos, el objetivo fundamental de dichos profesionales debería ser el de maximizar el valor del producto de una forma sostenible, es decir, minimizando sus impactos negativos (3).

Los estudios de arquitectura juegan, en este sentido, un papel fundamental, ya que tienen como objetivo realizar labores de diseño en el sector de la construcción, sector que destaca por el elevado impacto ambiental que genera.

A este respecto, cabe destacar como principales impactos el sobreconsumo de agua, materiales y energía, y las aguas residuales, residuos y emisiones generados (4). Concretamente, la construcción urbana representa en torno al $60 \%$ de las extracciones de materia prima de la litosfera y en España representa en torno al $12 \%$ del consumo total de agua (4). Asimismo, la construcción en España llega a generar cerca de una tonelada de residuos por habitante y año, del que, según el informe Symonds \& Ass, tan solo se recicla el $5 \%$ y el resto, a pesar de tener un gran potencial de reciclabilidad, va a parar a vertedero $(5,6)$. Además, casi el $50 \%$ de las emisiones de $\mathrm{CO}_{2}$ que se emiten a la atmósfera tiene relación directa con la construcción y uso de edificios, con la consecuente incidencia sobre el cambio climático (4).
Así las cosas, en todos los ámbitos del sector de la construcción en general, y en el de los estudios de arquitectura en particular, se hace necesario el desarrollo sistemas de gestión, formados por un conjunto interrelacionado de elementos (métodos, procedimientos, instrucciones, etc.), mediante los que la organización planifica y/o ejecuta y/o controla determinadas actividades relacionadas con los objetivos prefijados, que utilicen herramientas y estrategias para reducir estos impactos negativos $(1,7)$. Dentro de estas herramientas y estrategias, los estándares de ecodiseño se pueden considerar como una de las herramientas generales más reseñables (8).

En relación a los estándares más específicos del sector, ISO y CEN han desarrollado una serie de estándares destinados a controlar la sostenibilidad ambiental del sector de la construcción que se resumen en la Tabla 1.

Por otra parte, en el sector existen, además, herramientas específicas. Entre ellas, se pueden destacar:

- La metodología VERDE que permite evaluar la sostenibilidad de un edificio teniendo en cuenta aspectos regionales o locales, utilizando la técnica de adición de impactos de una forma objetiva (9).

- Los modelos destinados a reducir fuentes de impacto determinadas como los modelos destinados a reducir el consumo de energía (10).

- Las declaraciones ambientales de producto (EPD) que muestran información cuantitativa sobre el perfil ambiental del producto basada en indicadores de impacto ambiental. Estas declaraciones se basan en estudios de Análisis de Ciclo de Vida (ACV) elaborados por la empresa y que pueden ser verificados por una tercera parte independiente (11).

- Los sellos asociados a la norma de ecodiseño. Entre estas herramientas, el ECOPROYECTO permite al redactor del proyecto crear un distintivo basado en una declaración en el que muestra cómo el proyecto ha superado unos valores mínimos de ponderación de los impactos asociados a los aspectos ambientales (12).

Tabla 1. Normas ambientales elaboradas por ISO y CEN orientadas al sector de la construcción

\begin{tabular}{|l|l|}
\hline NORMA & OBJETO \\
\hline ISO/FDIS 15392 (13) & Principios generales de la sostenibilidad en la construcción. \\
\hline ISO/TS $21929(14)$ & Panel de indicadores destinados a medir la sostenibilidad de edificios. \\
\hline ISO $21930(15)$ & Principios para realizar una declaración ambiental de los edificios. \\
\hline PREN $15804(16)$ & Principios para realizar una declaración ambiental de los e dificios. \\
\hline PREN $15643(17)$ & Integración de los impactos ambientales en el sector de la construcción. \\
\hline
\end{tabular}

Fuente: Elaboración propia a partir de información obtenida por ISO y CEN. 
Sin embargo, también se observa que, como sucede en otros sectores de actividad económica, una serie de estándares no específicos del sector de la construcción están teniendo una fuerte influencia en él, debido a que en los últimos años hemos sido testigos de una aceleración del proceso general de estandarización, en un ámbito económico general caracterizado por la globalización y la integración de mercados (18). Así, y en lo que respecta a la gestión medioambiental, destaca el papel desarrollado por la familia de normas ISO 14000 (19). En esta línea, en el campo del ecodiseño debemos destacar la norma ISO 14062 que describe el proceso de integración de aspectos ambientales en el proceso de diseño y desarrollo de productos $(8,20)$.

Con estos antecedentes, en España, en junio de 2003, la Asociación Española de Normalización y Certificación (AENOR) decidió dar un paso más en esta línea y publicar una norma de Ecodiseño, la norma UNE $150301(1,6)$. Se ha de tener en cuenta, a modo de antecedente importante, que tanto las instituciones públicas españolas, como las empresas privadas, han experimentado un importante crecimiento en la adopción de distintos estándares de gestión medioambiental, como, por ejemplo, el estándar ISO 14001. Tal es así que, en la actualidad, España es el tercer país con mayor número de certificaciones conforme a la norma ISO 14001, y el primero en términos relativizados con respecto a su dimensión económica (21).

La referida norma UNE 150301 va más allá de otras normas medioambientales como la norma ISO 14040 (22), que se utilizan para analizar el ciclo de vida del producto, o la citada norma ISO/TR 14062 (20), ya que tiene por objeto proporcionar a las organizaciones los elementos de un Sistema de Gestión Medioambiental para que el proceso de diseño y desarrollo de productos y/o servicios sea efectivo, también desde el punto de vista ambiental (1).

Además, se ha de destacar el hecho de que esta norma española ha servido como base para la creación de la norma de ecodiseño ISO 14006:2011 (23). Teniendo en cuenta estos aspectos y la difusión que ha tenido este estándar en el sector de la construcción, el objetivo de este artículo consiste en analizar cómo han adoptado los estudios de arquitectura españoles el estándar UNE 150301. Concretamente, se trata de analizar cuáles fueron las motivaciones, los obstáculos y los beneficios de dicha adopción, una cuestión no estudiada previamente en la literatura.

\section{LA NORMA ESPAÑOLA UNE 150301}

La norma UNE 150301 fue promulgada por AENOR, que, asimismo, contó para su promoción con la colaboración de IHOBE, Sociedad pública de gestión ambiental del Gobierno Vasco. Estos dos organismos se encuentran representados en el comité técnico ISO/TC 207 encargado de elaborar el estándar ISO 14006.

Su objeto es especificar los requisitos del proceso de diseño y desarrollo de los productos y/o servicios de una organización que capaciten a ésta para establecer una sistemática de mejora continua de sus productos y/o servicios desde el diseño y desarrollo a través de un sistema de gestión. Su aplicación se centra en aquellos aspectos ambientales generados por los productos y/o servicios de la organización que ésta pueda controlar o sobre los que puede esperarse que tenga influencia.

La norma UNE 150301 se encuentra dividida en cuatro apartados principales. Los tres primeros son, al igual que en otras normas, los puntos clásicos relativos al "Objeto y campo de aplicación" -1-, "Normas para consulta" -2- y "Definiciones" -3-. El apartado 4, denominado "Requisitos del Sistema de Gestión Ambiental del Proceso de Diseño y Desarrollo" es donde se desarrolla el ciclo de mejora continua de esta norma (Figura 1). Este apartado se encuentra subdividido en seis puntos principales. En el primero de ellos, "Requisitos generales" -4.1 -, se remarca cómo la organización debe establecer y mantener al día estos requisitos definidos en función del alcance de la Gestión Ambiental del Proceso de Diseño y Desarrollo de Productos y/o servicios.

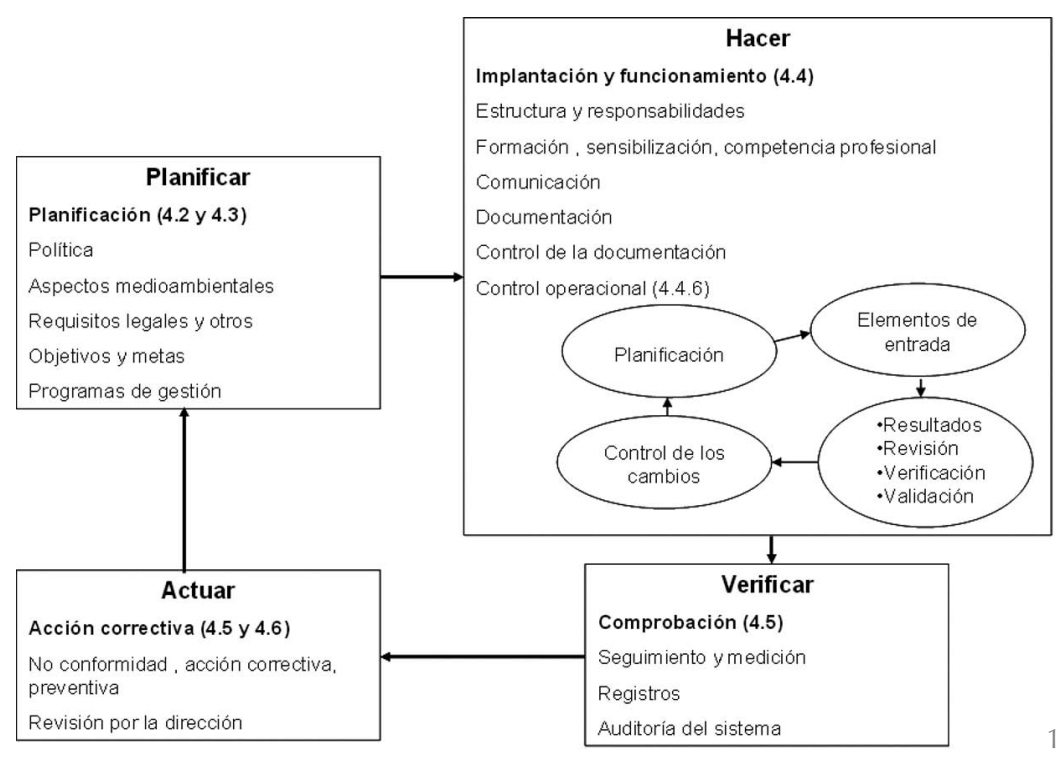

Fuente: Elaboración propia a partir de la norma UNE 150301
1. Proceso de mejora continua de la norma UNE 150301

(1)


El tercer punto hace referencia a la "Planificación" -4.3-. En este sentido, se comienzan "Identificando y analizando los aspectos ambientales de los productos de la organización" -4.3.1-, es decir, los elementos que producen impactos ambientales. Este estudio se realizará desde dos perspectivas diferentes en función de si se rediseñan productos ya existentes o si se diseñan nuevos. Teniendo en cuenta estos aspectos y "Los requisitos legales y otros requisitos del producto" -4.3.2- a nivel ambiental, la organización establecerá sus "Objetivos y metas" -4.3.3ambientales, consecuentes con la política del Sistema de Gestión Ambiental definida en el apartado 4.2 denominado "Política ambiental referente", y los documentará. A partir de estos objetivos, la organización desarrollará un "Programa de Gestión Ambiental del Proceso de Diseño y Desarrollo del Producto" -4.3.4-. En este programa se debe establecer la relevancia de los objetivos y las responsabilidades, los medios a emplear y el calendario en que han de ser alcanzados.

Posteriormente, se comenzará la "Implantación y funcionamiento" -4.4- del Sistema de Gestión Ambiental del Proceso de Diseño y Desarrollo. En él, se describen seis elementos fundamentales. El primero se refiere a la "Estructura y responsabilidades" -4.4.1-. Entre otros aspectos deben quedar claras las responsabilidades referidas a asegurar el cumplimiento de los requisitos del Sistema de Gestión Ambiental del Proceso de Diseño y Desarrollo y de informar de su funcionamiento. Asimismo, dentro del proceso de implantación se debe acometer la "Formación, sensibilización y competencia profesional" -4.4.2- del personal involucrado, en base a una adecuada educación, formación o experiencia apropiadas. Por otra parte, los tres siguientes subapartados inciden en la eficiencia de los procesos de "Comunicación" -4.4.3- tanto internos como externos, la "Documentación del Sistema de Gestión Ambiental del Proceso de Diseño y Desarrollo" -4.4.4- y el "Control de la documentación" -4.4.5-

Para finalizar el apartado de "Implantación y funcionamiento" -4.4-, el estándar remarca la importancia del "Control operacional" -4.4.6- para asegurar que las actividades del Proceso de Diseño y Desarrollo se efectúan bajo las condiciones especificadas; este control se subdivide en 7 subapartados: -4.4.6.1 - "Planificación del diseño y desarrollo", -4.4.6.2- "Elementos de entrada para el diseño", -4.4.6.3- "Resultados de diseño y desarrollo", -4.4.6.4- "Revisión del diseño y desarrollo], -4.4.6.5- "Verificación del diseño y desarrollo", -4.4.6.6- "Validación del diseño y desarrollo" y -4.4.6.7- "Control de los cambios del diseño y desarrollo".
Una vez puesto en funcionamiento el proceso, el apartado de "Comprobación y acción correctiva" -4.5- cierra el ciclo de mejora continua. El último punto, la "Revisión por la dirección" -4.6-, es común a otros Sistemas de Gestión, como los basados en la norma de Gestión de Calidad ISO 9001 y en la de Gestión Ambiental ISO 14001.

Una de las principales características de la norma UNE 150301 es que es certificable, al igual que el estándar ISO 14001, y a diferencia de otras normas como la ISO 14040 e ISO/TR 14062 que no son certificables (24). Además, resulta fácilmente integrable con las normas ISO 14001 e ISO 9001; de hecho en los anexos de la norma se explica cómo realizar la integración (1).

En lo que respecta a su difusión, cabe señalar que en España en noviembre de 2011 había al menos 129 empresas certificadas. Destacan como principales organismos certificadores AENOR con 67 certificaciones, el Instituto de la Construcción de Castilla y León (ICCL) con 33 y Applus+ con 15 certificados emitidos.

En cuanto a la distribución sectorial, es destacable que pese a que en un primer momento la norma estaba enfocada al sector industrial, con el paso del tiempo se constata una mayor difusión entre los estudios de arquitectura (25). Concretamente, en esta fecha el $73 \%$ de las empresas, 94 de las 129 empresas, que habían adoptado esta norma eran estudios de arquitectura (Figura 2). Además, se comprueba que 114 de las 129 empresas certificadas se encuentran relacionadas directa o indirectamente con el sector de la construcción, ya que, además de los 94 estudios de arquitectura, están certificadas 11 empresas que diseñan muebles, 5 que diseñan electrodomésticos, 2 ascensores, otra material para la instalación eléctrica en residencial y terciario y otra materiales de construcción derivados de la madera.

Al analizar la distribución geográfica, hemos observado la gran cantidad de empresas certificadas existentes en la CAPV, en donde se concentran 57 de las 129 adopciones de las que 39 son estudios de arquitectura. Una de las posibles razones de esta concentración es el esfuerzo que están realizando las instituciones vascas con objeto de promocionar la adopción de estándares de gestión. Concretamente, en el sector de la construcción este impulso se realiza a través del programa Eraikal, programa que subvenciona el 50\% de los costes de consultoría y auditoría que tienen las empresas del sector durante el proceso (26). Además, tal como se muestra en la Figura 2, destaca la existencia de 17 estudios certificados en la Comunidad Autónoma de 
Cataluña, 13 en la Comunidad Foral de Navarra y 12 en la Comunidad de Madrid.

\section{METODOLOGÍA}

Con el objetivo de analizar las motivaciones, dificultades y resultados de la adopción de la norma UNE 150301 por los estudios de arquitectura, se diseñó un estudio empírico exploratorio basado en metodología cualitativa. Esta metodología fue seleccionada debido a que se adecua al análisis del complejo proceso de adopción del estándar UNE 150301. En la planificación de una investigación de naturaleza descriptiva, principalmente exploratoria, esta metodología permite profundizar más en el proceso y obtener una mejor comprensión de la naturaleza a estudiar $(27,28)$.

En concreto, se seleccionaron cinco estudios innovadores en la implantación de Sistemas de Gestión y que habían sido certificados en una primera fase, anterior a 2008, con objeto de que ya tuvieran cierta experiencia en trabajar utilizando la norma UNE 150301. Además, influyeron otros aspectos como su ubicación geográfica y su disponibilidad para participar en la investigación. Las empresas elegidas fueron Oneka Arquitectura, Ramón Ruíz Cuevas Arquitectos, Ache Arquitectura, Gausark y Toledo Taldea para desarrollar un estudio en profundidad de casos.

El estudio se desarrolló entre enero de 2009 y setiembre de 2010 y consistió en una secuencia de entrevistas en profundidad semi-estructuradas con directivos de las cinco organizaciones siguiendo un guión semi-flexible con el mismo número de preguntas y con un protocolo de evaluación de los casos en función de cada factor para asegurar la fiabilidad (29). Asimismo, se siguieron las pautas marcadas en la literatura para garantizar la validez y la fiabilidad del estudio. Sin embargo, en este artículo sólo se han desarrollado tres de los casos analizados, ya que se obtuvo un nivel de saturación alto como consecuencia de que los procesos seguidos y los resultados obtenidos fueron muy similares. Sin embargo, las conclusiones consignadas se han generalizado a los cinco estudios de casos.

\section{ANÁLISIS DE CASOS}

\subsection{Gausark}

La empresa Gausark es un estudio de arquitectura que cuenta con 11 trabajadores que desarrollan actividades de diseño, desarrollo y seguimiento de la ejecución de proyectos de edificación, urbanización, rehabilitación, derribo, urbanismo, seguridad y salud, y programas de control de calidad, entre otras. En el año 2001 adoptó ISO 9001 debido a motivaciones de tipo

\begin{tabular}{|l|c|c|}
\hline COMUNIDAD & TOTAL & $\begin{array}{c}\text { ESTUDIOS DE } \\
\text { ARQUITECTURA }\end{array}$ \\
\hline País Vasco & 57 & 39 \\
\hline Cataluña & 18 & 17 \\
\hline Navarra & 18 & 13 \\
\hline Madrid & 14 & 12 \\
\hline Castilla y León & 9 & 4 \\
\hline La Rioja & 5 & 4 \\
\hline $\begin{array}{l}\text { Comunidad } \\
\text { Valenciana }\end{array}$ & 3 & 0 \\
\hline $\begin{array}{l}\text { Principado } \\
\text { de Asturias }\end{array}$ & 1 & 1 \\
\hline $\begin{array}{l}\text { Castilla } \\
\text { La Mancha }\end{array}$ & 1 & 1 \\
\hline Cantabria & 1 & 1 \\
\hline Andalucía & 1 & 1 \\
\hline Islas Canaria & 1 & 1 \\
\hline Total & $\mathbf{1 2 9}$ & $\mathbf{9 4}$ \\
\hline
\end{tabular}

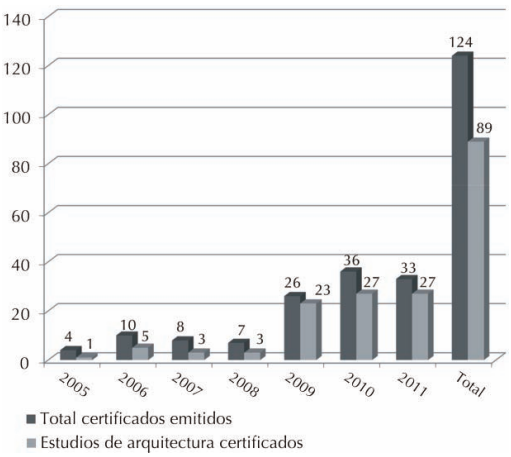

Fuente: Elaboración propia a partir de datos proporcionados por AENOR en setiembre de 2011.

interno. Más adelante, en el año 2007, implantaron la norma de Gestión Ambiental ISO 14001. En este caso, la motivación principal fue externa ya que se preveía que cada vez se iba a tener más en cuenta en los concursos públicos de proyectos.

Al empezar la implantación de la norma de Gestión Ambiental ISO 14001 la consultora les recomendó adoptarla junto con el estándar de Ecodiseño UNE 150301:2003, ya que éste también iba a ser muy valorado en dichos concursos que, con la amenaza de la crisis, eran cada vez más competitivos.

Asimismo, otros factores importantes fueron las subvenciones existentes para la implantación de estos sistemas a través del programa Eraikal, la sensibilidad que existía en la empresa hacia los aspectos ambientales, y que el impacto ambiental que producen sus diseños es muy superior a los residuos generados por el estudio.

La implantación se realizó integrándola en el antiguo manual de calidad basado en la norma de Gestión de Calidad ISO 9001. En aquel entonces, el manual de calidad constabade 18 procedimientos a los que se añadió dos nuevos procedimientos: uno referente a la adopción de la ISO 14001 y el último referente a la UNE 150301. La elaboración de este último procedimiento fue mucho más laboriosa ya que los aspectos ambientales relacionados con el producto y los impactos ambientales que producen son grandes y difíciles de cuantificar.

De cara a elaborar esta documentación, se tomó como referencia la norma UNE 150301, la experiencia que tenía el estudio en proyectos anteriores y un proyecto de 196 viviendas de protección oficial a ejecutar en suelo urbano. El ciclo de vida de sus diseños se subdividió en 4 fases: Extracción y fabricación de productos utilizados, Ejecución del edificio, De-construcción del edificio y Uso y mantenimiento del edificio construido. 
Posteriormente, se empezaron a analizar los aspectos ambientales que influían en cada una de las fases y se elaboró un panel de indicadores para medir el impacto ambiental de éstos. Esta fase fue de gran complejidad ya que, tal como se observa en la Tabla 2, era necesario tener en cuenta la actividad desarrollada por sus proveedores en el proceso de extracción, fabricación y transporte de los productos utilizados, la actividad realizada en el proceso de ejecución, cómo se realizaría la deconstrucción, así como, el uso y mantenimiento que se le iba a dar. En todas estas actividades, intervienen numerosos agentes que el estudio no puede controlar, si bien, el diseño que realiza es clave en el impacto producido en cada fase.

La adopción se realizó junto con el diseño de 196 viviendas protección oficial. Para ello, en la primera fase, la fase de anteproyecto además de realizar un análisis del programa de necesidades y un análisis del planeamiento y normativas a cumplir, se analizaron los aspectos ambientales en cada una de las fases del ciclo de vida del producto. En ella se realizó la propuesta de medidas ambientales y se marcaron los objetivos ambientales a cumplir. Concretamente, en esta fase se plantearon 41 propuestas de las que el cliente rechazo 22 , principalmente, debido a aspectos económicos y a la imposibilidad de modificar planeamientos y pliegos. Estas medidas estaban relacionadas fundamentalmente con los siguientes aspectos ambientales:

- Reducir el consumo energético.

- Reducir el consumo de agua.

- Minimizar las emisiones atmosféricas.

- Minimizar la generación de residuos.

Posteriormente, en la fase proyecto básico se adaptaron al proyecto las 28 medidas aceptadas por el cliente y se modificaron las propuestas que no habían sido aceptadas. De estas modificaciones, se aceptaron tres relativas a la reducción de emisiones atmosféricas en la fase de ejecución. Concretamente, estaban relacionadas con el riego del terreno para evitar la generación de

Tabla 2. Principales aspectos ambientales en cada etapa del ciclo de vida

\begin{tabular}{|l|l|l|}
\hline ETAPA & ASPECTOS SIGNIFICATIVOS & OTROS ASPECTOS \\
\hline $\begin{array}{l}\text { Extracción y } \\
\text { fabricación }\end{array}$ & $\begin{array}{l}\text { Consumo de materias primas } \\
\text { Consumo de energía }\end{array}$ & $\begin{array}{l}\text { Consumo de agua } \\
\text { Generación de residuos } \\
\text { Emisión de ruidos }\end{array}$ \\
\hline del edifición & $\begin{array}{l}\text { Consumo de energía } \\
\text { Emisiones atmosféricas }\end{array}$ & $\begin{array}{l}\text { Generación de residuos } \\
\text { Vertidos al agua } \\
\text { Emisiones de ruidos } \\
\text { Biodiversidad }\end{array}$ \\
\hline $\begin{array}{l}\text { Uso y } \\
\text { mantenimiento }\end{array}$ & $\begin{array}{l}\text { Consumo de energía } \\
\text { Consumo de agua } \\
\text { Emisiones atmosféricas } \\
\text { Impacto visual } \\
\text { Uso del suelo }\end{array}$ & $\begin{array}{l}\text { Emisiones atmosféricas } \\
\text { Emisión de ruidos }\end{array}$ \\
\hline Deconstrucción & $\begin{array}{l}\text { Consumo de energía } \\
\text { Generación de residuos }\end{array}$ & \\
\hline
\end{tabular}

Fuente: Elaboración propia a partir de la información aportada por la empresa. polvo, con la forma de retirar los sobrantes y escombros a la finalización de los trabajos y con evitar las operaciones de pintado con pistola in situ. Por último, en el proyecto de ejecución se adaptaron estas nuevas medidas aceptadas al proyecto y se realizó una verificación de las medidas con estructuras e instalaciones. En la Tabla 3, se muestran en letras de color negro las propuestas que derivaron en acciones ejecutadas con sus resultados agrupados por fase y en letras color gris las propuestas que fueron rechazadas.

Una vez finalizado el proceso se elaboró el libro del edificio. En él se detalla el plan de mantenimiento del edificio. Este aspecto es esencial ya que esta etapa es la de mayor duración y en ella el impacto ambiental que se produce es muy importante. En el proceso de adopción de la norma se estableció que además de dar las indicaciones para mantener y desarrollar las medidas ambientales del edificio, se debería intentar fomentar individualmente buenas prácticas ambientales.

Una vez desarrollado este proceso se procedió a elaborar nueva documentación para implementar esta forma de trabajo y utilizar la misma metodología en futuros proyectos.

En el proceso de adopción de esta metodología las principales dificultades que se han encontrado tanto en este proyecto como en otros, están relacionadas con los siguientes aspectos:

- Materiales de construcción. Se trata de un sector en desarrollo en el que la documentación de los nuevos productos es escasa y los precios no son competitivos. De hecho, pese a que en este proyecto el cliente había aceptado el uso de materiales con etiquetado ecológico, en el proyecto de ejecución se rechazó esta medida por estos motivos.

- Económicas. Las soluciones novedosas tienen un coste poco competitivo. Especialmente, en las promociones de VPO se hace extremadamente difícil implementarlas debido a que en estos tiempos se trabaja con márgenes escasos.

- Normativa: La necesidad de aprovechar al máximo la edificabilidad, el Código Técnico de Edificación y las limitaciones del planeamiento y programa de necesidades del cliente restan flexibilidad para implementar ciertas medidas.

La adopción de la UNE 150301 les ha supuesto una gran satisfacción ya que consideran que los resultados han sido buenos, pese a que el cliente no aceptó el 39\% de las medidas propuestas. 
En la Tabla 3 se muestra un resumen de las actuaciones realizadas y los resultados obtenidos en comparación con los previstos inicialmente en cada una de las fases del ciclo de vida del producto. Dentro de estos resultados destacan la reducción de las emisiones generadas, del consumo de agua y, principalmente, del consumo de energía en todas las fases del ciclo de vida.

Además, piensan que los verdaderos beneficios están por llegar, ya que el ecodiseño va a adquirir mayor importancia en el sector de la construcción, debido a entre otros aspectos, en muchos casos les permite adaptarse previamente a algunos cambios que se producen en el sector.

En relación a la integración señalan que la implantación de las normas ISO 14001 y la UNE 150301 las han realizado integrándolas junto con el manual de calidad basado en la norma de Gestión de Calidad ISO 9001. Sin embargo, esta integración no ha provocado grandes cambios en el manual y no les ha supuesto gran esfuerzo ya que las propias normas detallan la forma en que se deben integrar.

Tabla 3. Resumen de las principales propuestas, acciones y resultados obtenidos

\begin{tabular}{|c|c|c|}
\hline ETAPA & PROPUESTAS Y ACCIONES REALIZADAS & RESULTADOS \\
\hline $\begin{array}{l}\text { Ejecución } \\
\text { del edificio }\end{array}$ & $\begin{array}{l}\text { - Utilización de materiales con etiquetado ecológico } \\
\text { - Utilización de materiales reciclados } \\
\text { - Utilización de materiales reciclables a su fin de vida } \\
\text { - Utilización de materiales libres de productos tóxicos y contaminantes } \\
\text { - Diseño que facilite nuevas instalaciones futuras } \\
\text { - Uso de materiales de gran durabilidad bajo consumo energético }\end{array}$ & $\begin{array}{l}\text { Reducción } \\
\text { de materiales } 50 \% \\
\text { Ahorro de energía } 5 \%\end{array}$ \\
\hline $\begin{array}{l}\text { Ejecución } \\
\text { del edificio }\end{array}$ & $\begin{array}{l}\text { - Eliminación de grupos electrógenos de obra } \\
\text { - Riego del terreno para evitar la generación de polvo } \\
\text { - Retirada de los escombros a la finalización de los trabajos } \\
\text { - Evitar el pintado con pistola in situ }\end{array}$ & $\begin{array}{l}\text { Ahorro } \\
\text { de energía } 80 \% \\
\text { Reducción } \\
\text { de emisiones } 30 \%\end{array}$ \\
\hline $\begin{array}{l}\text { Uso y } \\
\text { mantenimiento }\end{array}$ & 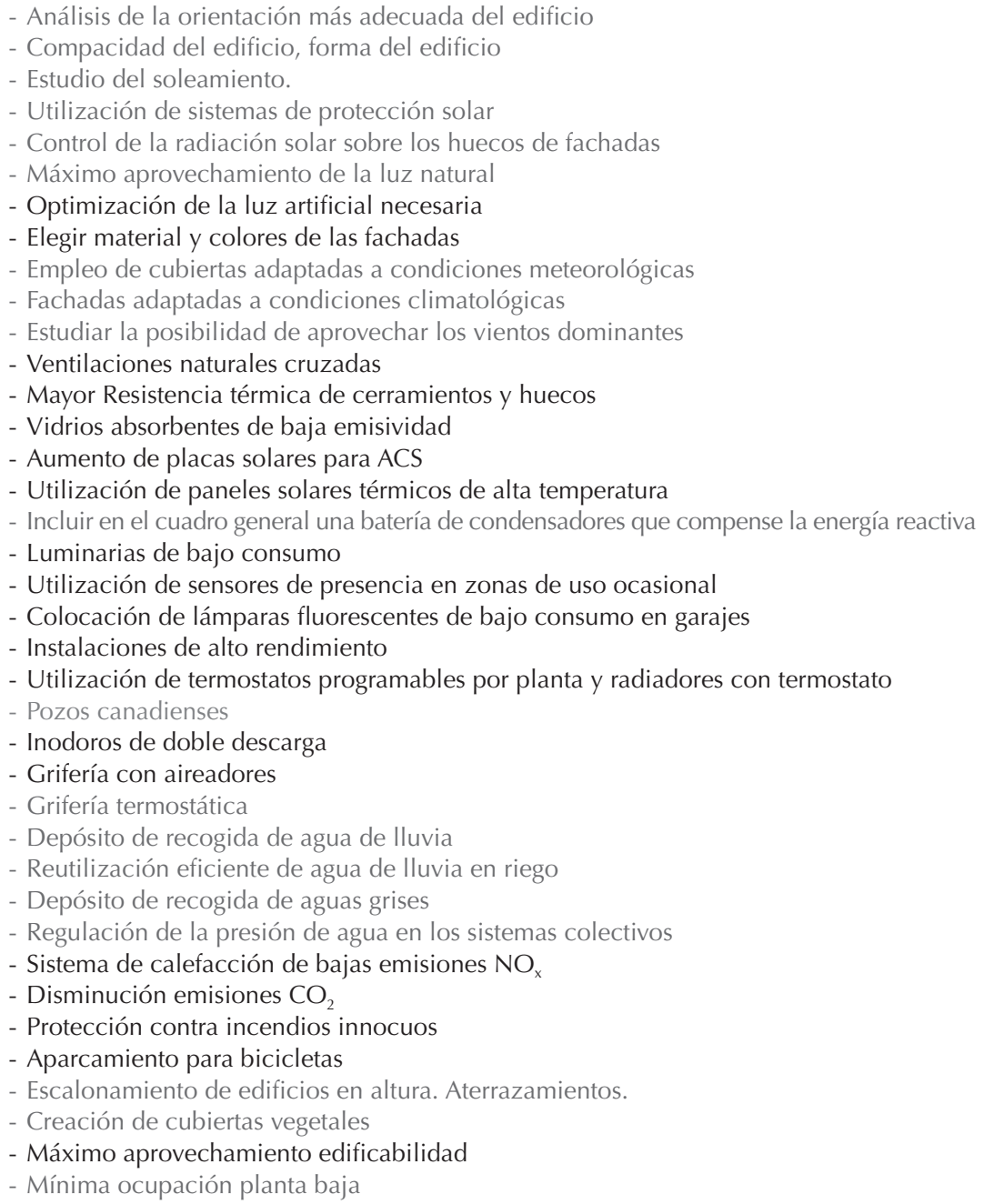 & $\begin{array}{l}\text { Ahorro } \\
\text { de energía 20\% } \\
\text { Ahorro } \\
\text { de agua } 30 \% \\
\text { Reducción } \\
\text { de emisiones 20\% }\end{array}$ \\
\hline
\end{tabular}

Fuente: Elaboración propia a partir de la información aportada por la empresa.

Nota: Las propuestas realizadas se han marcado en fuente negra y las rechazadas en fuente gris. 


\subsection{Toledo Taldea}

Toledo Taldea es un estudio de arquitectura que se encuentra dividido en cuatro secciones Edificación, Urbanismo, Ordenación del territorio y Asesoría y que cuenta con 15 trabajadores.

Esta empresa implantó de forma integrada un sistema basado en la norma de Gestión de Calidad ISO 9001, la norma de Gestión Ambiental ISO 14001 y la de Ecodiseño UNE 150301 en el año 2006, si bien el alcance de esta última sólo afecta a la sección de edificación.

La motivación principal que les llevó a implantar la UNE 150301 fue un intento de mejorar su capacidad comercial, ya que existen determinados concursos donde este certificado se valora mucho. De forma previa a la adopción de la UNE 150301, implantaron el estándar ISO 14001 que no les ha supuesto un gran esfuerzo ya que el estudio no tiene grandes consumos ni genera muchos residuos, y les ha servido como herramienta para adoptar la norma de ecodiseño. Sin embargo, el proceso de adopción de la UNE 150301 fue para ellos un reto muy laborioso, ya que el producto que diseñan genera un gran impacto ambiental a lo largo de su ciclo de vida, y a que su diseño y las medidas ambientales propuestas deben ser aprobados por el cliente.

El proyecto que seleccionaron para adoptar la norma UNE 150301 fue un pabellón industrial destinado a centralizar los vehículos y las oficinas de una mancomunidad. Una vez analizadas las necesidades del proyecto, tal como se recoge en la Tabla 4, se identificaron los aspectos ambientales que debían ser tenidos en cuenta durante el desarrollo del proyecto. Además, se comprobó que los impactos ambientales más importantes se daban en la fase de uso y mantenimiento y eran debidos al consumo de agua y energía.

Para medir los impactos ambientales se desarrolló un panel de indicadores que tenía como objetivo poder cuantificar el grado de mejora ambiental que se obtendría si se implantaba cada una de las medidas propuestas.

Tabla 4. Principales aspectos ambientales en cada etapa del ciclo de vida

\begin{tabular}{|l|l|}
\hline ETAPA & ASPECTOS SIGNIFICATIVOS \\
\hline Extracción y fabricación & Consumo de materias primas \\
\hline Ejecución del edificio & $\begin{array}{l}\text { Consumo de energía } \\
\text { Generación de residuos }\end{array}$ \\
\hline Uso y mantenimiento & $\begin{array}{l}\text { Consumo de energía } \\
\text { Consumo de agua } \\
\text { Generación de residuos }\end{array}$ \\
\hline Deconstrucción & Generación de residuos \\
\hline
\end{tabular}

Fuente: Elaboración propia a partir de la información aportada por la empresa.
En relación al consumo energético, se comenzó analizando las necesidades mínimas para desarrollar la actividad en el edificio teniendo en cuenta que la energía más limpia y menos contaminante es la energía ahorrada.

Con este objetivo, se realizó un estudio de los condicionantes externos al pabellón que podían tener influencia en la demanda final, como son el microclima de la parcela y la evolución de las sombras a lo largo del día. Posteriormente, con objeto de reducir la demanda se diseñó la distribución y orientación del edificio. Se orientaron las estancias de trabajo continuado al sureste y las de paso, almacén o utilización ocasional de personas al norte. Además, se diseñó la fachada sureste con grandes ventanales y con un doble cierre que tenían como objeto aprovechar la luz natural y mantener la temperatura del edificio.

Una vez minimizada la energía consumida, se trataron de encontrar medios de generación y aprovechamiento de energía limpia. Entre ellos cabe destacar la utilización de:

- Paneles solares fotovoltáicos destinados a generar energía eléctrica.

- Un sistema de climatización por fancoils, que regula una caldera de condensación de máximo rendimiento que trabaja con temperaturas de impulsión bajas, y que se apoya en paneles solares térmicos para minimizar su consumo, y una máquina de absorción para generar frío aprovechando el calor producido por los paneles solares térmicos.

El segundo objetivo era reducir el consumo de agua. Para ello, se diseñó un sistema doble de recogida de agua que constaba, por un lado, de un sistema de recogida con capacidad para recoger $170 \mathrm{~m}^{3}$ de aguas de Iluvia proveniente de un área de $2.000 \mathrm{~m}^{2}$ de la parcela y por otro, de un sistema de aprovechamiento del agua utilizada en duchas y lavabos. Esta agua es aprovechada para llenar las cisternas de los baños y para la limpieza de contenedores y camiones.

La empresa considera que la implantación de la norma ha sido un reto muy interesante del que se han obtenido buenos resultados que se muestran de forma resumida en la Tabla 5. Concretamente, en relación a las previsiones iniciales han conseguido reducir el consumo energético del edificio en un $45 \%$, principalmente gracias a la máquina de absorción que permite ahorrar un $18 \%$, a los paneles térmicos que aportan un $14 \%$ de la energía consumida y a la caldera de condensación que supone un ahorro del $10 \%$. Por otra parte, la recogida de agua de 
Tabla 5. Resumen de las principales acciones y resultados obtenidos

\begin{tabular}{|l|l|l|}
\hline ETAPA & ACCIONES & RESULTADOS \\
\hline Extracción y fabricación & Utilización de materiales de obra con doble función. & Reducción de materiales 5\% \\
\hline Ejecución del edificio & Utilización de materiales preindustrializados. & $\begin{array}{l}\text { Ahorro de energía } 15 \% \\
\text { Ahorro agua } 70 \%\end{array}$ \\
\hline Uso y mantenimiento & $\begin{array}{l}\text { Paneles solares fotovoltáicos } \\
\text { Sistema de climatización por fancoils } \\
\text { Caldera de condensación de máximo rendimiento } \\
\text { Paneles solares térmicos } \\
\text { Máquina de absorción para generar frío } \\
\text { Recogida de agua de lluvia. } \\
\text { Reaprovechamiento del agua de duchas y lavabos. } \\
\text { Eliminar uso de aceites en maquinaria ascensor }\end{array}$ & $\begin{array}{l}\text { Ahorro de energía } 45 \% \\
\text { Ahorro de agua } 75 \% \\
\text { Reducción consumo aceite ascensor 100\% }\end{array}$ \\
\hline Deconstrucción & Utilización de materiales reciclables & Reaprovechamiento materiales part. Int. 90\% \\
\hline
\end{tabular}

Fuente: Elaboración propia a partir de la información aportada por la empresa.

lluvia y el reaprovechamiento del agua de duchas y lavabos permiten reducir el consumo de agua en un $75 \%$.

Además, consideran que la adopción del estándar les ha ayudado a mejorar su imagen y la de sus diseños, así como a mejorar la reflexión de su proceso creativo. Pese a todo, esperaban mejores resultados, especialmente en relación al esfuerzo realizado. En este sentido, señalan que el problema que tienen los estudios de arquitectura es que al final las decisiones de ecodiseño que suponen un aumento de coste no suelen ser aprobadas por los promotores, ya que el cliente final no valora lo suficiente el ecodiseño para asumir la subida del precio, especialmente ahora en estos tiempos de crisis.

\subsection{Oneka arquitectura}

Oneka arquitectura, S.L. es un estudio de arquitectura que cuenta con seis empleados y que realiza sus diseños utilizando una metodología que tiene como principales puntos de referencia la satisfacción de sus clientes, así como la del resto de partícipes sociales y cumplir los principios de la organización basados principalmente en aspectos ambientales, económicos y sociales.

En el año 2002, adoptó la Norma de Gestión de Calidad ISO 9001: 2000 y desde esa fecha empezó a trabajar en la mejora de aspectos ambientales, principalmente en mejorar la eficiencia energética de sus diseños. Posteriormente, en 2005 adoptó la norma ISO 14001: 2004. Sin embargo, al observar que el impacto ambiental de sus diseños era mucho mayor que el del propio estudio decidieron adoptar la normativa de ecodiseño UNE 150301: 2003 que la integraron en su sistema de gestión.

La principal motivación para adoptar la norma UNE 150301 fue interna ya que los aspectos ambientales se recogían como un factor clave a mejorar en su sistema de ges- tión. Asimismo, la aparición de normativas específicas en materia ambiental, la inclusión de criterios ambientales en normativas existentes, como el Código Técnico de la Edificación, y unas experiencias previas en las que habían obtenido buenos resultados fueron otros factores que sirvieron para reafirmar la importancia que tenía para la empresa la adopción de una metodología de ecodiseño.

El proyecto seleccionado para la adopción de la norma de ecodiseño tenía como finalidad rehabilitar y ampliar una antigua fábrica de Harina de Bilbao y acondicionarla para albergar un museo y estancias del departamento de Salud y Consumo del ayuntamiento. Una vez analizadas las posibilidades de la parcela y las necesidades del proyecto, se realizó una identificación de los aspectos ambientales del mismo, analizando todas las fases de su ciclo de vida. El resultado de este análisis, que se muestra en la Tabla 6, dio como aspectos más significativos el consumo de energía del edificio, ocasionada principalmente por las necesidades de calefacción y refrigeración, y el ruido generado al convivir en el edificio 2 tipos de actividades muy diferentes, la de oficinas y la museística, a las que hay que unir una estación cercana de ferrocarril.

Tabla 6. Principales aspectos ambientales en cada etapa del ciclo de vida

\begin{tabular}{|l|l|}
\hline \multicolumn{1}{|c|}{ ETAPA } & ASPECTOS SIGNIFICATIVOS \\
\hline Extracción y fabricación & Consumo de materias primas \\
\hline Ejecución del edificio & $\begin{array}{l}\text { Consumo de materias primas } \\
\text { Toxicidad de materiales }\end{array}$ \\
\hline \multirow{2}{*}{ Uso y mantenimiento } & $\begin{array}{l}\text { Consumo de energía } \\
\text { Consumo de agua } \\
\text { Generación de ruido }\end{array}$ \\
\hline Deconstrucción & Generación de residuos \\
\hline
\end{tabular}

Fuente: Elaboración propia a partir de la información aportada por la empresa

Posteriormente, se comenzó un proceso de generación de ideas para solucionar, o al menos minimizar, la incidencia de dichos aspectos ambientales. En esta etapa del proyecto, la empresa utilizó como herramienta 
la Guía de Edificación Sostenible (30). De entre todas las medidas posibles, el equipo de trabajo valoró y seleccionó las más adecuadas, teniendo en cuenta la viabilidad técnica, la económica y la ambiental.

Estas medidas combinaban una serie de medidas activas y pasivas, que se muestran de forma resumida en la Tabla 7 . Entre ellas, destaca el diseño de una envolvente alrededor del edificio con un sistema de capas múltiples que contribuye a reducir la entrada de ruido del exterior, aumentar la inercia térmica y el aislamiento, especialmente, en las zonas con orientaciones más desfavorables. Otra mejora importante, se logró mediante la instalación de sistemas pasivos de captación y sistemas de control solar, con persianas de lamas orientables. Por otra parte, se decidió cambiar las ventanas de acero existentes, por otras de aluminio con rotura de puente térmico y doble acristalamiento de baja emisividad.

En relación a los sistemas de aporte energético, se decidió instalar calderas de condensación de máxima eficiencia que aprovechan el calor latente de vaporización del agua contenida en los gases de combustión y lo traspasan al agua de entrada. Estas calderas se ubicaron en dos puntos centrados en el edificio con objeto de minimizar los recorridos de las conducciones y maximizar el rendimiento global.

Por otra parte, para reducir el ruido interior, además de la envolvente exterior, se instalaron techos especialmente acústicos y trasdosados de lana de roca.
La aplicación de las medidas planteadas ha supuesto una mejora general del comportamiento ambiental del edificio. Las previsiones iniciales de consumo energético se han reducido en un $29 \%$, principalmente, gracias a la utilización de calderas de condensación y a la envolvente exterior. La reducción del nivel de ruido también ha sido significativa reduciéndose en $45 \mathrm{~dB}$ el proveniente del exterior y en $10 \mathrm{~dB}$ el producido por las bajantes que eran las principales fuentes internas de ruido. Además, se ha logrado reducir el consumo de agua en un $37 \%$ gracias a la utilización de aireadores. Por último, se ha reducido de forma significativa la utilización de materiales y la generación de residuos, gracias entre otros aspectos a, haber aprovechado la fachada antigua, no haber utilizado materiales pesados ni disolventes y haber instalado una tabiquería interior desmontable que se puede reutilizar en el futuro.

\section{CONCLUSIONES}

El gran impacto ambiental producido por el sector de la construcción hace necesaria la adopción de medidas eficaces que ayuden a reducir dicho impacto. Además de las normas y regulaciones de obligado cumplimiento que se van estableciendo con el paso del tiempo, desde algunas administraciones se está impulsando la adopción en el sector de estándares técnicos y de gestión específicos orientados a mejorar el conocimiento, el control y la reducción del impacto ambiental.

Entre los estándares de gestión señalados cabe referirse a los estándares de ecodiseño,

Tabla 7. Resumen de las principales acciones y resultados obtenidos

\begin{tabular}{|c|c|c|}
\hline ETAPA & ACCIONES & RESULTADOS \\
\hline Extracción y fabricación & - Aislamientos en base a corcho aglomerado & Reducción materiales pesados $100 \%$ \\
\hline Ejecución del edificio & $\begin{array}{l}\text { - Conservación de la fachada Antigua } \\
\text { - Pinturas de silicato } \\
\text { - No se utilizan disolventes orgánicos } \\
\text { - Utilización de polibutileno en conducciones }\end{array}$ & $\begin{array}{l}\text { Reducción consumo materiales } 70 \% \\
\text { Se elimina el minio } \\
\text { Eliminación de soldaduras }\end{array}$ \\
\hline Uso y mantenimiento & $\begin{array}{l}\text { - Envolvente del propio edificio } \\
\text { - Sistemas pasivos de captación energética } \\
\text { - Calderas de condensación de máxima eficiencia } \\
\text { - Ventanas con doble acristalamiento y rotura de Puente térmico } \\
\text { - Sistema de control solar con lamas orientables } \\
\text { - Puntos centrados de producción de calor y frío } \\
\text { - Sistemas de iluminación de bajo consumo con } \\
\text { sistemas de detección de presencia } \\
\text { - Trasdosado de aislamiento de lana de roca } \\
\text { - Techo especialmente acústico } \\
\text { - Paneles acústicos } \\
\text { - Reducción de presión de agua } \\
\text { - Utilización de grifos aireadores } \\
\text { - Inodoros de doble descarga } \\
\text { - Sistema de recogida de agua de lluvia }\end{array}$ & $\begin{array}{l}\text { Reducción del consumo energético } 29 \% \\
\text { Reducción ruido del exterior } 45 \mathrm{~dB}(\mathrm{~A}) \\
\text { Reducción ruido del interior } 10 \mathrm{db}(\mathrm{A}) \\
\text { Reducción del consumo agua } 37 \%\end{array}$ \\
\hline Deconstrucción & $\begin{array}{l}\text { - Láminas de caucho EPDM } \\
\text { - Conducciones polibutileno, polipropileno y poliolefinas } \\
\text { - Tabiquerías interiores desmontables } \\
\text { - Perfilería de aluminio }\end{array}$ & Reducción de la generación de residuos $45 \%$ \\
\hline
\end{tabular}


como la norma UNE 150301, que son de gran ayuda para sistematizar acciones que tienen como objetivo controlar y generar medidas para reducir el impacto ambiental del producto a lo largo de las distintas fases del ciclo de vida (31). Ésta es una de las razones principales que ha llevado al comité ISO/TC 207 a aprobar en julio de 2011 la norma de ecodiseño ISO 14006, que utiliza como referencia el estándar UNE 150301.

En esta investigación se ha analizado la implantación de la norma UNE 150301 en cinco estudios de arquitectura. En lo que respecta a la gestión, los cinco estudios tienen características similares. En todos los casos, las empresas están certificadas conforme a la norma de Gestión de Calidad ISO 9001 y en 3 conforme a la normativa de Gestión Ambiental ISO 14001. Además, a cuatro de los cinco estudios se les puede considerar pioneros en este aspecto ya que han implantado sus estándares de gestión en etapas muy tempranas. Además, consideran que la adopción previa de estos estándares, especialmente la norma de Gestión Ambiental ISO 14001, ha facilitado la de la UNE 150301.

Las tres empresas que han implantado el estándar ISO 14001 consideran que la adopción de la norma de Ecodiseño UNE 150301 les ha supuesto mucho más esfuerzo, aunque han obtenido unos resultados significativamente mejores, ya que el impacto ambiental directo que produce un estudio de arquitectura no es en absoluto comparable al de sus diseños. Además, señalan que pese a no lograr que el cliente aprobase todas sus propuestas, han conseguido reducciones de impacto significativas principalmente debido a que han conseguido reducir la energía consumida en todas las fases del ciclo de vida.

Si analizamos el proceso, podemos señalar que las empresas comienzan realizando un diagnóstico del impacto ambiental a través de un panel de indicadores. Conforme a este diagnostico, incorporan al proyecto un conjunto de procesos de mejora continua destinados a minimizar el impacto ambiental.

Una de las principales dificultades que señalan está relacionada con los materiales de construcción. El hecho de que en España apenas existen Declaraciones Ambientales de Producto (EDP), la escasa documentación relativa a aspectos ambientales que presentan los fabricantes y en muchos casos la falta de competitividad que tienen en aspectos técnicos y económicos han limitado los resultados ambientales de la adopción.

Sin embargo, en todos los casos señalan que uno de los principales problemas que han tenido está relacionado con la aprobación por parte del cliente de las medidas para reducir el impacto ambiental que suponen un aumento de costes que, normalmente, suelen ser rechazadas aunque en muchos casos supongan una reducción de costes en la fase de uso. Este hecho coincide con resultados obtenidos en otros estudios, que incluso señalan que en muchos casos la reducción del impacto ambiental suele ser un aspecto valorado negativamente por el cliente (32).

Para evitar estos problemas, las administraciones juegan un papel fundamental a la hora de establecer normas de obligado cumplimiento y de valorar los aspectos ambientales en las licitaciones públicas. En este sentido, en los últimos años se han aprobado numerosas normas que afectan directa o indirectamente al sector de la construcción y que sirven para reducir el impacto ambiental de los diseños, no sólo en la fase edificación, sino a lo largo de todo el ciclo de vida. Sin embargo, en relación a las demandas que realizan determinados sectores de la sociedad, en algunos casos las administraciones, en especial en tiempos de crisis económica como los que se viven en la actualidad, actúan demasiado tarde a la hora de implantar normativas. Este retraso en el sector de la construcción causa costes directos de implantación que restan competitividad a las empresas respetuosas con el medio ambiente, así como costes indirectos de adecuación y uso a los usuarios finales de edificios poco sostenibles.

Por último, señalar que los estudios se encuentran satisfechos con la adopción. Algunos destacan que les ha ayudado a realizar una reflexión profunda de su proceso creativo. Otros indican que ha contribuido para mejorar su imagen y su posición en concursos. Asimismo, señalan que el proceso de mejora continua les ha permitido reducir costes de la fase de edificación y principalmente a través de la optimización del consumo de agua y energía de la fase de uso. Por todos estos motivos, existe acuerdo entre los estudios a la hora de señalar que les ha servido para mejorar su posición competitiva, pese a que algunos de ellos, en relación al esfuerzo realizado esperaban mejores resultados.

\section{AGRADECIMIENTOS}

Este artículo se ha realizado en el marco del Grupo de Investigación del Sistema Universitario Vasco IT423-10, financiado por el Gobierno Vasco. Los autores desean agradecer a las distintas empresas, instituciones y personas que han colaborado activamente en el estudio realizado. Asimismo, los autores desean agradecer las sugerencias y comentarios realizados por los revisores anónimos de la revista. 


\section{BIBLIOGRAFÍA}

(1) UNE 150301:2003: Gestión ambiental del proceso de diseño y desarrollo, Ecodiseño, AENOR, Madrid, 2003.

(2) Brutland, G.: "Our common future", Report of the World Commission on Environment and Development, New York, 1987.

(3) Hamza, N.; Horne, M. : "Educating the designer: An operational model for visualizing low-energy architecture", Building and Environment, vol. 42 n 11 (2007), pp. 3841-3847.

(4) Marti i Rague, X.: "Edificación sostenible", X Congreso nacional de medioambiente CONAMA. Madrid, 2010.

(5) Del Río, M.; Izquierdo, P.; Salto, I.; Santa Cruz, J.: "La regulación jurídica de los residuos de construcción demolición (RCD) en España. El caso de la Comunidad de Madrid", Informes de la Construcción, vol. 62, no 517 (2010), pp. 81-86.

(6) IHOBE: Guías sectoriales de ecodiseño, IHOBE, Bilbao, 2010.

(7) IHOBE: Guía para el desarrollo de la norma de Ecodiseño UNE 150301:2003 (Base de ISO 14006). Evaluación de Aspectos Ambientales de Producto, IHOBE, Sociedad Pública de Gestión Ambiental, Bilbao, 2010.

(8) Arana, G.; Heras, I.: "Paving the way for the ISO 14006 ecodesign standard: an exploratory study in Spanish companies", Journal of Cleaner Production, vol. 19, no 9 (2011), pp. 1007-1015.

(9) Macías, M.; García Navarro, J.: "Metodología y herramienta VERDE para la evaluación de la sostenibilidad en edificios", Informes de la Construcción, vol. 62, nº 517 (2010), pp. 87-100.

(10) Batlle, T.; Pich-Aguilera, F.: "Revitalización de antiguos cuarteles a partir de un nuevo espacio climático. Criterios para la reducción de la demanda energética. Parque Científico Tecnológico Agroalimentario 'PCiTAL'. Gardeny. Lleida. Consorcio 'PCital' (Univ. Lérida-Ayuntamiento Lérida)", Informes de la Construcción, vol. 62, no 517 (2010), pp. 113-124.

(11) Arboix, J.: "EPD’s de materiales de construcción", Congreso Regional Internacional Sustainable Building, SB10mad. Madrid, 2010.

(12) Instituto de la Construcción de Castilla y León: Innovación y sostenibilidad en el sector de la construcción, Foro regional sobre sostenibilidad e I+D+I, Valladolid, 2011.

(13) ISO/FDIS 15392:2008: Sustainability in building construction - General Principles, ISO, Geneva, 2008.

(14) ISO/TS 21929:2006: Sustainability in building construction - Sustainability indicators- Framework for the development of indicators for buildings, ISO, Geneva, 2006.

(15) ISO 21930:2007: Sustainability in building construction - Environmental declaration of building products, ISO, Geneva, 2007.

(16) prEN 15804:2008: Sustainability of construction works - Environmental product declarations - Product Category Rules, CEN, Bruselas, 2008.

(17) prEN 15643:2008: Sustainability of construction works - Integrated assessment of building performance, CEN, Bruselas, 2008.

(18) Heras, I.: ISO 14001 y otros estándares de gestión: pasado, presente y futuro, Editorial Civitas. Madrid, 2006

(19) ISO 14001:2004: Environmental management systems - Requirements with guidance for use, ISO. Geneva, 2002

(20) ISO/TR14062:2002: Environmental management - Integrating environmental aspects into product design and development. British Standards Institution, London, 2002.

(21) Heras, I.; Arana, G.; Díaz de Junguitu, A.; Espí, M. T.; Molina, J. F.: Los Sistemas de Gestión Medioambiental y la competitividad de las empresas de la CAPV, Instituto Vasco de Competitividad. Bilbao, 2008.

(22) ISO 14040:2006: Environmental management-Life cycle assessment - Principles and Framework, ISO, Geneva, 2006.

(23) ISO 14006:2011: Environmental management systems - Guidelines for incorporating ecodesign. ISO, Geneva, 2011.

(24) IHOBE: De la UNE 150.301 a la ISO 14006: Gestión ambiental del proceso de diseño y de desarrollo, III Encuentro de empresas en Ecoinnovación, IHOBE, Bilbao, 2008.

(25) Arana, G.; Heras, I.: Adopción de la norma de UNE 150301 de Ecodiseño. Un estudio de casos, DYNA Ingeniería e industria, vol. 85, nº 8 (2010), pp. 652-661.

(26) Eraikal: Programa para la promoción de la implantación y de la mejora de los SGs de la calidad, fomento de la sostenibilidad y apoyo a la innovación en las empresas del sector de la edificación residencial de la CAPV, Departamento de vivienda y asuntos sociales, Vitoria, 2010.

(27) Eisenhardt, K.: "Building theories from case study research", Academy of Management Review, vol. 14, no 4 (1989), pp. 532-550.

(28) Yin, R. K.: "Case Study Research: Design and Methods", Thousand Oaks, Sage Publications, California, 2003

(29) Maxwell, J.: "Qualitative Research Design. An Interactive Approach", Thousand Oaks, Sage Publications, California, 2005.

(30) EVE, IHOBE, ORUBIDE VISESA, Departamento de Vivienda y Asuntos Sociales del Gobierno Vasco: Guía de Edificación Sostenible para la Vivienda en la Comunidad Autónoma del País Vasco, Bilbao, 2006.

(31) Arana, G.; Heras, I.; Cilleruelo, E.: "A case study of the adoption of a reference standard for ISO 14006 in the lift industry", Clean Technologies and Environmental Policy. Doi: 10.1007/s10098011-0427-4, pp.1-9.

(32) Luttropp, C.; Lagerstedt, J.: “Ecodesign and the Ten Golden Rules: generic advice for merging environmental aspects into product development", Journal of Cleaner Production, vol. 14, $\mathrm{n}^{\circ} 15$ (2006), pp.1396-1408. 